\title{
Studies on the Time of Valvular Movements in Mitral Valvular Disease with Ultrasonic Doppler Method
}

\author{
Tsuneo YOSHIDA, M.D., * Masayoshi MORI, M.D., Yasuharu NIMURA, \\ M.D., Shinpachi TAKAGISHI, M.D., and Katsumi \\ NAKANISHI, M.D.
}

The application of ultrasound to the examination within the body furnishes one with a tactile information which would be otherwise impossible. Especially the application of ultrasonic Doppler method assures one of reliable data for valvular movements which bear an influential, physiological and clinical significance.

The aim of the present study was directed to the detection with ultrasonic Doppler method of the time of valvular movements in cases of mitral valvular disease. The findings obtained here are very likely to promise to afford fundamental understanding in interpreting various pathophysiological entities.

$\mathrm{D}^{\mathrm{s}}$

ETERMINATION of the time of valvular movements of the heart offers fundamental understanding in analysing the motion of the heart and interpreting the significance of heart sounds and murmurs. Clinically, a socalled opening snap and Hegglin syndrome constitute a concept closely related to the valvular movements. The time of valvular movements has been analysed mainly on the basis of pressure curve, pulse curve, electrokymogram, phonocardiogram, etc. But these analytical methods are indirect ones, being not without some technical shortcomings.

With its sharp directivity and transmittivity in tissues, ultrasound enables one to perform detailed examinations within the body. Especially the ultrasonic Doppler method can detect the time of valvular movements. ${ }^{1) \rightarrow 4}$ As an application of the ultrasonic Doppler method, determination of the time of valvular movements in mitral valvular disease is being reported.

\section{MetHods}

Application of the Doppler Method on the Heart-

When a continuous ultrasonic beam, $3 \mathrm{Mc} / \mathrm{s}, 20 \sim 50 \mathrm{~mW} / \mathrm{cm}^{2}{ }^{2}$ is transmitted ${ }^{1)}$, 2) to the heart from an electrosonic transducer which is placed on the chest wall (Fig. 1), part of which transmitted is reflected from the tissue boundary such as the free wall, septum or valves of the heart. The reflection of ultrasound in the heart is mainly carried out on the endocardial surface, i.e., the blood-tissue boundary, ${ }^{5)}$ where exists a discontinuity of sonic impedance values. The reflected wave, being sub-

From the First Department of Medicine, Osaka University Medical School, Osaka (Director: Prof. Tsuneo YOSHIDA).

* Professor of Medicine. 


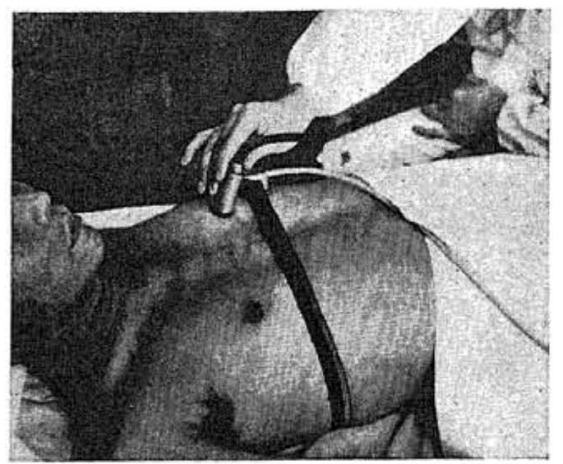

Fig. 1. A transducer is presented being manipulated.

ject to Doppler effect due to the movements of the target, shows a slight difference in frequency from its primary one. Therefore, if both reflected wave and direct wave are simultaneously detected with the same transducer, there appears between these waves a beat having a frequency which is proportionate to the velocity of the target. Namely, if the velocity of the target is $u$, the wave length of ultrasound in the body tissue $\lambda$, the angle between the direction of the beam and the velocity of the target $\alpha$ and the frequency of the beat $f_{d}$, there follows

$$
\mathrm{f}_{\mathrm{d}}=\frac{2 \mathrm{u}}{i} \cos \alpha
$$

On the basis of the beat, it is able to obtain information about the existence of the movements of the target and the velocity.

The apparatus consists of a high-frequency oscillator, an electrosonic transducer made of barium titanate, a detector, an amplifier, filters and a recorder (Fig. 2). The detailed description of the apparatus have been published in elsewhere. ${ }^{1-4)}$ The Doppler signals obtained are recorded on the electromagnetic oscillogram with ECG and PCG simultaneously. The paper speed used in this

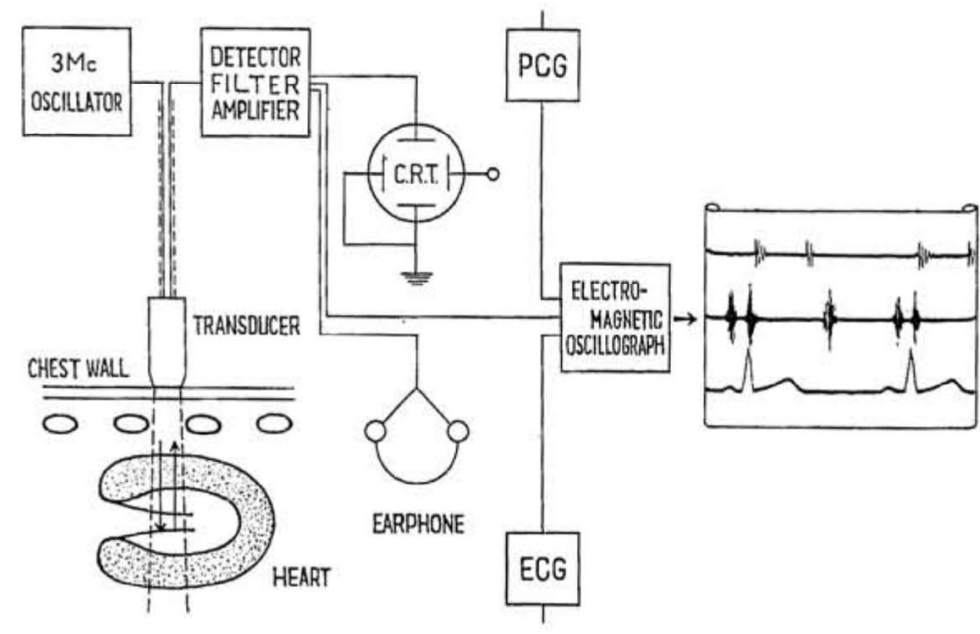

Fig. 2. Schematic block diagram of the instrument. 
study was about $20 \mathrm{~cm}$./sec. As monitor, a Braun tube and an earphone were employed.

Doppler signals were recorded on oscillopaper as vibrations. When the target stands still there appear no signals, and the curve regains the base line. If the direction of the target's movement is at a right angle to the direction of ultrasonic beam there appear no signals. But, practically all the signals could be obtained since the target was not a smooth plane.

Of the Doppler signals obtained with this method ${ }^{11}$ those which are related with the heart wall have a frequency of about $200 \mathrm{c} / \mathrm{s}$, and those which are due to valvular movements have a frequency of about $1000 \mathrm{c} / \mathrm{s}$. These 2 kinds of signal are separated by means of filters and individually recorded.

The Doppler signals due to valvular movements (Fig. 3a, 3b, 3c, 3d) have a short duration; they appear in early systole and early diastole being related with the opening and closing of the valves.
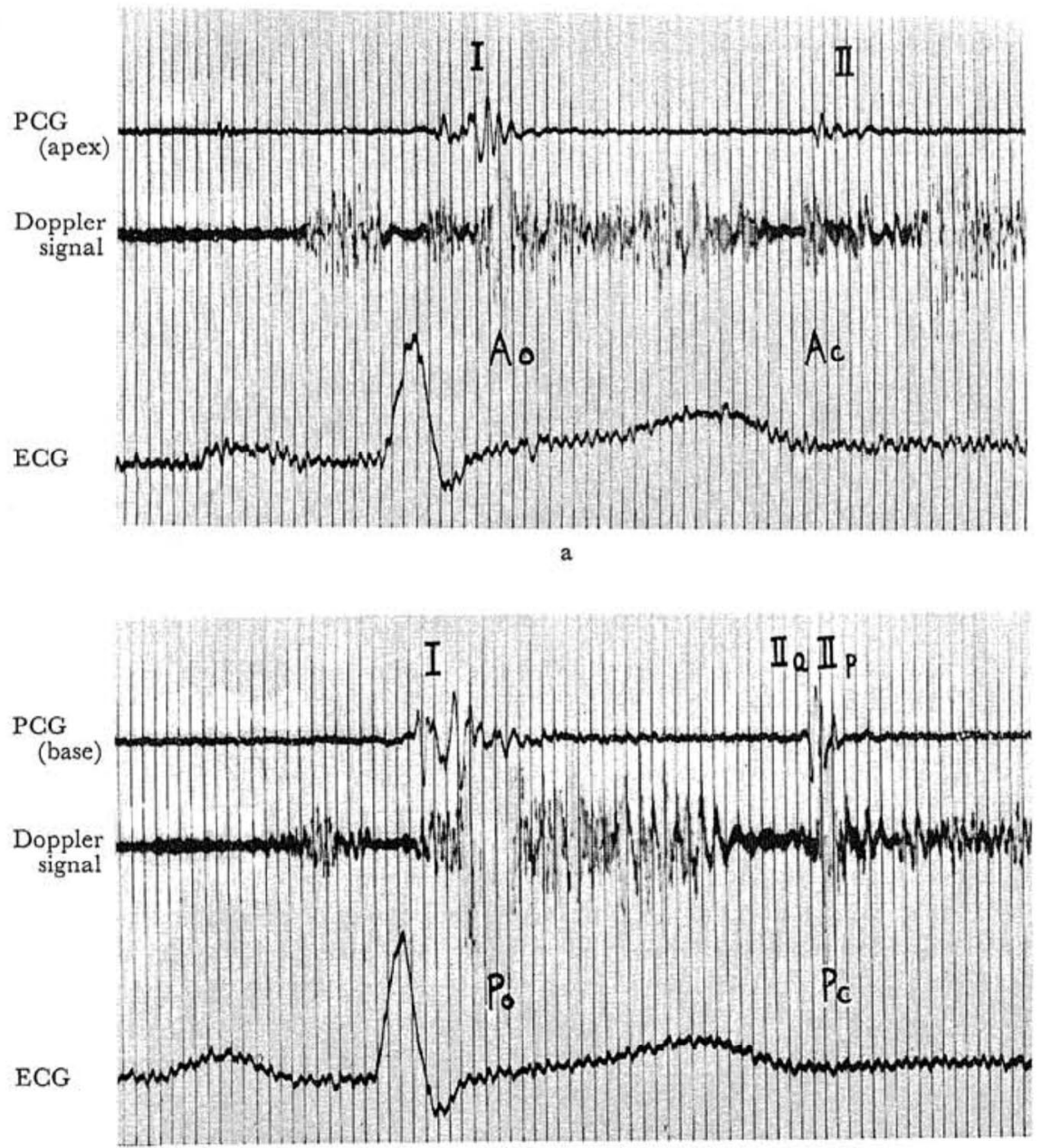

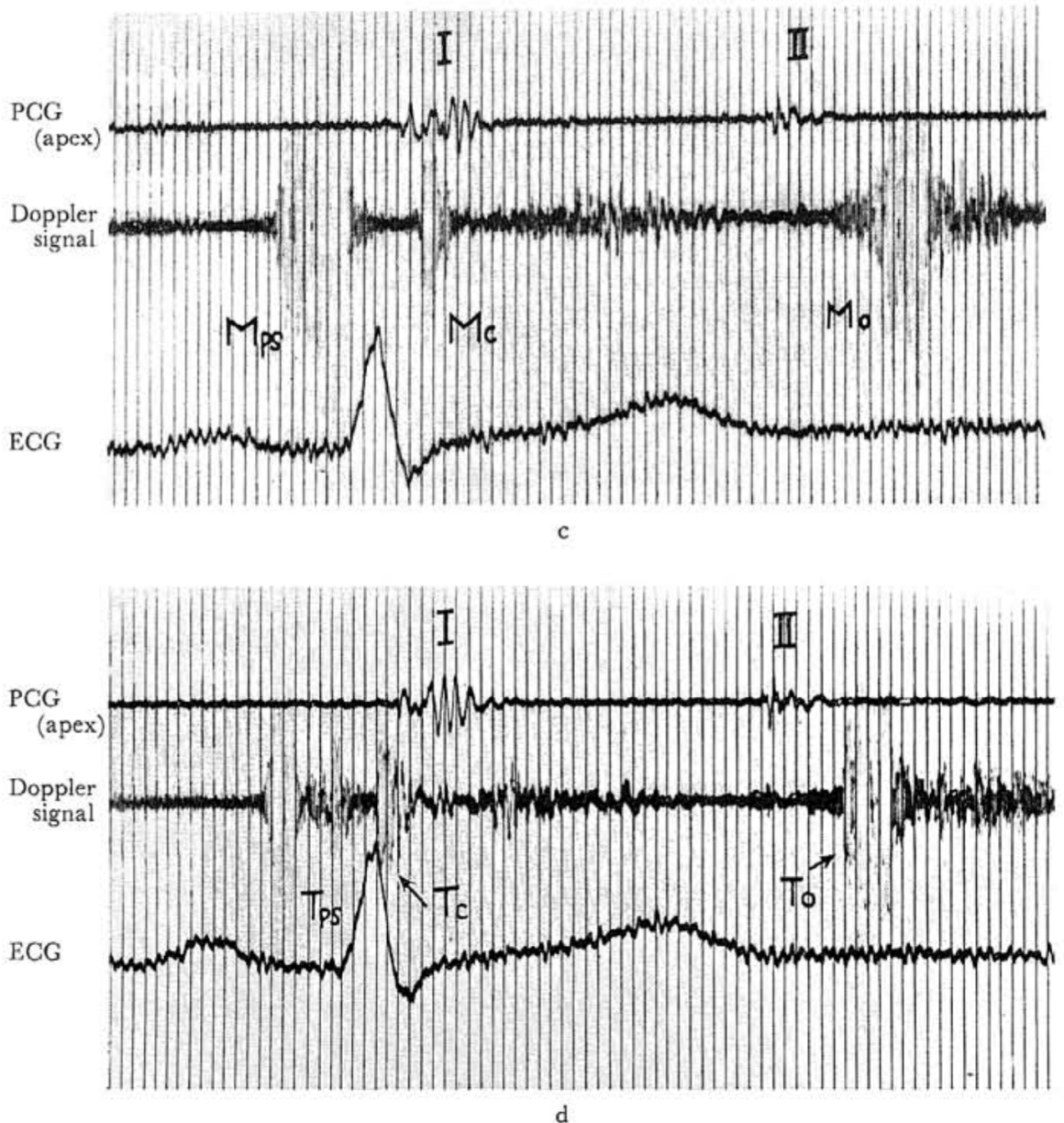

Fig. 3. Ultrasonic Doppler signal interpreted to be related with valves.

a. aortic valve. Ao: opening. Ac: closing.

b. pulmonic valve. Po: opening. Pc: closing.

c. mitral valve. Mc: closing. Mo: opening.

d. tricuspid valve. Tc: closing. To: opening.

(S.N., a 34-year-old healthy man)

It has been established that the area to place an electrosonic transducer for the respective valves was inductively determined on the basis of the anatomical location of the valves, the findings of such cases as bundle branch block in which the time of valvular movements in the left heart would be different from that in the right heart, as well as on the findings in other pathological conditions. $\left.{ }^{1,}, 6\right)$

For instance, near the left sternal border in the third or fourth intercostal space is selected for the aortic valve; near the left sternal edge in the second or third intercostal space for the pulmonary valve; near the left parasternal line in the fourth intercostal space for the mitral valve; near the left sternal border in the fourth or fifth intercostal space for the tricuspid valve. 
Since the areas indicated for the respective valves are partially superimposed, sometimes it is difficult to decide whether the Doppler signals obtained belong to which semilunar valve or which atrioventricular valve.

A signal indicating the opening of the aortic valve (Ao) appears in early systole; a signal indicating the closure of it ( $\Lambda c$ ) appears just before the second heart sound (Fig. 3a). Likewise, regarding the pulmonary valve, signals Po and Pc appear corresponding to the opening and closure of it (Fig. $3 \mathrm{~b})$. The end of a signal indicating the closure of the semilunar valve coincides with the beginning of the second heart sound. ${ }^{1,7}$. When the times of the closure for both semilunar valves are different and the second heart sound is split or reduplicated, there appear Ac and Pc prior to the components of the second heart sound, IIa and IIp, which are related to the aortic valve and the pulmonary valve respectivel $:, 1,7$

A signal indicating the closure of the mitral valve ( $\mathrm{Mc}$ ) appears in early systole; a signal indicating the opening of it (Mo) appears in early diastole (Fig. 3c). Likewise, signals $T c$ and $T_{0}$ appear corresponding to the closure and the opening of the tricuspid valve (Fig. 3d). Regarding to the mitral valve and tricuspid valve, besides the above mentioned signals, signals of about $1000 \mathrm{c} / \mathrm{s}$ in frequency $(\mathrm{Mps}$ and Tps respectively) begin to appear $0.10 \sim 0.13$ sec. after the beginning of $\mathrm{P}$ wave (Fig. $3 \mathrm{c}$ and $3 \mathrm{~d}$ ). The development of these signals seems attributable to a passive movement of the atrioventricular valve due to the impelling of the blood into the ventricle, being subject to the atrial contraction. ${ }^{11,31,6}$

The duration McAo between the beginning of the signal $\mathrm{Mc}$ and the signal Ao is defined as an isometric contraction of the left ventricle and the duration TcPo as that of the right vetricle; the duration AcMo is defincd as an isometric relaxation of the left ventricle and the duration PcTo as that of the right ventricle. However, it must be admitted that $\mathrm{Ac}_{\mathrm{c}}$ and $\mathrm{Pc}$, as mentioned above, kecp a certain time relationship with the second heart sound or IIa, IIp respectively. So, for the convenience, IIaMo, and IIpTo were employed instead of AcMo and PcTo respéctively in this paper.

Besides the aforesaid low-frequency and high-frequency Doppler signals, there was recorded during systole and diastole a noise which lasts long with a considerably high frequency..$^{2,4}$. This noise secms to be originated from disturbances in reflection coefficient of a medium on the path of ultrasound and in phase of reflected wave caused by a turbulance of the blood.

The advantages of this method are as follows:

1. The findings are free from a time lag.

2. The onset and completion of valvular movements are detected separately.

3. It is easy to detect the time of an atrioventricular valve opening. This time affords a maked variation under various conditions.

4. The operating procedure is simple.

It is assured that this method enables one to perform a time phase analysis of heart motion centering around the time of valvular movements, leading to an application on pathophysiologic and diagnostic researches.

\section{Materials}

Thirty cases of nearly compensated mitral stenosis and 22 cases of mitral 
stenosis and insufficiency with sinus rhythm were studied. The cases with atrial fibrillation were excluded from this stt... nce they were complicated with effects of arrhythmia.

A control group from the previous studies ${ }^{1,}, 6$ were used for the comparison.

\section{Results}

Closure of Atrioventricular Valve in Mitral Valvular Disease-

Signal Mps which appears following P-wave in healthy subjects does not appear in most cases of mitral valvular disease. All the 30 cases of mitral stenosis had no signal Mps (Fig. 4a) ; 22 cases of mitral stenosis and insufficiency
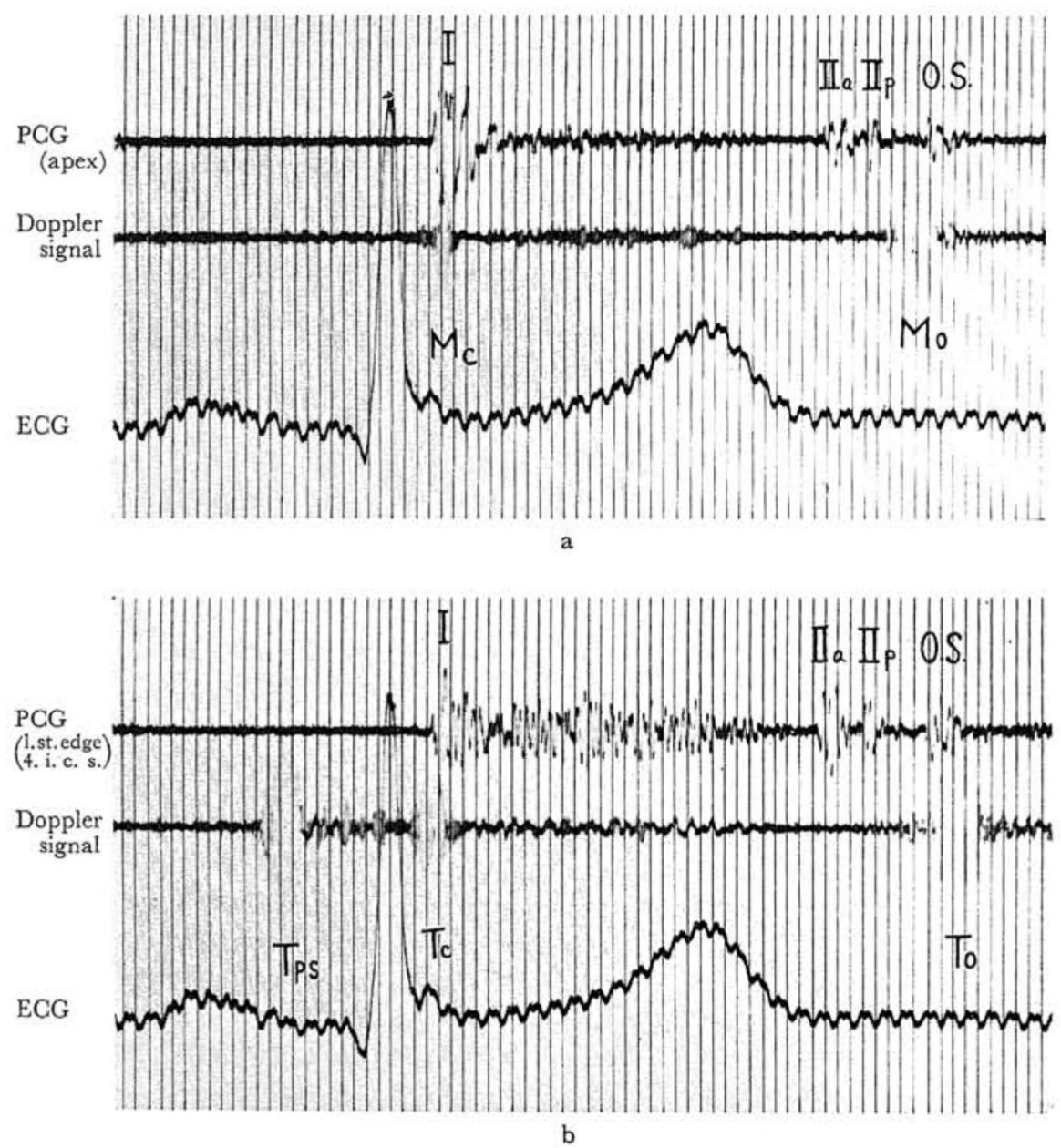

Fig. 4. Movements of atrioventricular valves in mitral valve disease. a, mitral valve. $b$, tricuspid valve. $M p s$ is absent. Tps appears. Tc precedes Mc. Mo precedes To. (T.M., a 26-year-old man with mitral stenosis) 
except for 3 cases, did not have the signal. All cases which demonstrated an identification of signals of tricuspid valve origin revealed Tps (Fig. 4b). It was suggested that in cases of mitral valvular disease the mitral valve was hardly moved when the blood was impelled into the left ventricle by means of atrial contraction. The hardness of the mitral valve is assumed to explain this phenomenon.

Cases of mitral valvular disease generally show a prolongation of QMc, i.e., the duration between the beginning of $\mathrm{QRS}$ and the beginning of signal Mc (Fig. 5), over healthy subjects. This prolongation expresses a delay in the closure of the mitral valve (Table I). This trend was markedly observed in cases of mitral stenosis.

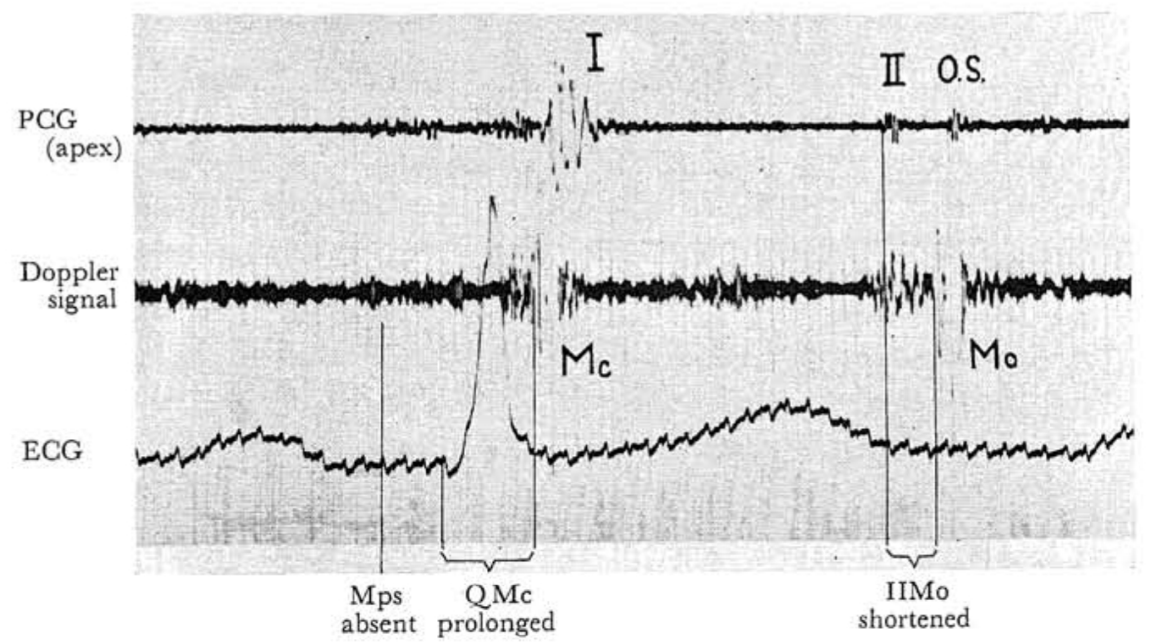

Fig. 5. Mitral valve movements in mitral stenosis. Mps is absent. Mc delayed; IIMo shortened. The opening snap begins while Mo develops. (H.Y., a 17-year-old girl with mitral stenosis)

In all cases where the signal of the mitral valve and that of the tricuspid valve were both identified the closing of the tricuspid valve preceded that of the mitral valve (Fig. 4a and 4b; Table II).

Opening of Atrioventricular Valve in Mitral Valvular Disease-

In cases of mitral valvular disease the opening of the mitral valve preceded that of the tricuspid valve. Cases with mitral valular disease showed a shortening of IIMo, i.e., an isometric relaxation of the left ventricle as compared with healthy subjects (Fig. 5 ; Table III). The second heart sound of cases of mitral valvular disease was often split or reduplicated. Such cases showed a shortening of IIaMo, i.e. the duration of an isometric relaxation of the left ventricle was shortened (Fig. 4a; Table II). IITo or IIpTo, i.e., an isometric relaxation of the right ventricle was not shortened (Fig. 4b; Table II).

Mitral opening snap starts in the latter half of the duration of an opening 
Table I. Time of Mitral Valve Closure in Cases of Mitral Valular Disease. MS: Mitral Stenosis MSI: Mitral Stenosis and Insufficiency

\begin{tabular}{c|c|c|c}
\hline QMc $\downarrow$ & MS & MSI & Healthy \\
\hline sec. $^{\text {sec }}$ & $0^{\text {cases }}$ & $0^{\text {cases }}$ & $1^{\text {cases }}$ \\
0.04 & 0 & 1 & 14 \\
0.05 & 0 & 4 & 28 \\
0.06 & 6 & 7 & 20 \\
0.07 & 9 & 3 & 10 \\
0.08 & 12 & 5 & 3 \\
0.09 & 1 & 0 & 0 \\
0.10 & 2 & 1 & 0 \\
& cases & 21 cases & cases \\
& 30 & & 76
\end{tabular}

Table II. Time of Atrioventricular Valve Movements in Cases Showing Identification of Signals of Mitral Valve and of Tricuspid Valve.

\begin{tabular}{|c|c|c|c|c|c|c|c|c|c|c|}
\hline Patient & Age & Sex & $\begin{array}{c}\text { Clinical } \\
\text { Diagnosis }\end{array}$ & QMc & QTc & $\begin{array}{l}\text { IIaMo } \\
\text { or } \\
\text { II Mo }\end{array}$ & $\begin{array}{l}\text { IIpTo } \\
\text { or } \\
\text { II To }\end{array}$ & $\begin{array}{l}\text { QIIa } \\
\text { or } \\
\text { QII }\end{array}$ & QIIp & QT \\
\hline S. K. & 26 & $f$ & MS & $\begin{array}{l}\text { sec. } \\
0.07\end{array}$ & $\begin{array}{r}\text { sec. } \\
0.05\end{array}$ & $\begin{array}{l}\text { sec. } \\
0.06\end{array}$ & $\begin{array}{r}\text { sec. } \\
0.05\end{array}$ & $\begin{array}{r}\text { sec. } \\
0.38\end{array}$ & $\begin{array}{l}\text { sec. } \\
0.41\end{array}$ & $\begin{array}{r}\text { sec. } \\
0.37\end{array}$ \\
\hline N.S. & 33 & $\mathrm{~m}$ & MS & 0.08 & 0.06 & 0.03 & 0.04 & 0.34 & 0.37 & 0.30 \\
\hline K. T. & 38 & $f$ & MS & 0.09 & 0.08 & 0.06 & 0.07 & 0.38 & 0.41 & 0.39 \\
\hline K. S. & 23 & $f$ & MS & 0.10 & 0.08 & 0.03 & 0.05 & 0.38 & - & 0.37 \\
\hline T. M. & 26 & $\mathrm{~m}$ & MS & 0.08 & 0.06 & 0.06 & 0.06 & 0.38 & 0.41 & 0.37 \\
\hline S. T. & 24 & $\mathrm{~m}$ & MS & 0.08 & 0.06 & 0.02 & 0.10 & 0.33 & - & 0.33 \\
\hline M. N. & 23 & f & MS & 0.08 & 0.07 & 0.04 & 0.06 & 0.42 & - & 0.45 \\
\hline H. G. & 19 & $f$ & MS & 0.06 & 0.55 & 0.06 & 0.07 & 0.39 & - & 0.38 \\
\hline K. H. & 37 & $\mathrm{~m}$ & MS & 0.07 & 0.06 & 0.06 & 0.06 & 0.39 & 0.43 & 0.37 \\
\hline Y. K. & 24 & $f$ & MSI & 0.06 & 0.05 & 0.04 & 0.06 & 0.40 & 0.42 & 0.39 \\
\hline S. M. & 27 & $\mathrm{~m}$ & MSI & 0.10 & 0.07 & 0.02 & 0.07 & 0.36 & - & 0.32 \\
\hline M. Y. & 41 & $f$ & MSI & 0.06 & 0.05 & 0.05 & 0.07 & 0.40 & - & 0.40 \\
\hline K. S. & 18 & $\mathrm{~m}$ & MSI & 0.05 & 0.04 & 0.05 & 0.07 & 0.39 & 0.42 & 0.41 \\
\hline M. K. & 23 & $f$ & MSI & 0.08 & 0.04 & 0.03 & 0.06 & 0.38 & - & 0.36 \\
\hline M. K. & 25 & $\mathrm{~m}$ & MSI & 0.08 & 0.06 & 0.06 & 0.07 & 0.41 & 0.43 & 0.41 \\
\hline N. I. & 21 & f & MSI & 0.06 & 0.04 & 0.05 & 0.07 & 0.42 & - & 0.42 \\
\hline Y. T. & 12 & f & MSI & 0.04 & - & 0.04 & 0.06 & 0.39 & - & 0.38 \\
\hline T. F. & 12 & $\mathrm{~m}$ & MSI & - & 0.03 & 0.04 & 0.06 & 0.35 & - & 0.34 \\
\hline
\end{tabular}

movement of the mitral valve (Fig. 4a, 5).

Opening of Semilunar Valve in Mitral Valvular Disease-

Generally speaking, it is occasionally difficult to differentiate the signal of the aortic valve opening, Ao, and that of the pulmonary valve opening, 
Po. ${ }^{1)}$ It scems because the detecting areas for these 2 are partially superimposed or the opening time for them is almost the same. In general Po seems more easily detectable than Ao.

These values of cases with mitral valvular disease which were considered to be QPo are shown in Table IV. Ordinarily they showed little difference from those of healthy subjects, but comparatively long values were observed in cases of mitral valvular disease. However, if Ao and Po are not both identified, there is a possibility of introduction of QAo due to the technical reason mentioned above.

In 7 cases, in which Ao and Po were both identified, Po showed a delay over Ao except for one case; QPo showed a trend of prolongation over healthy subjects (Fig. 6a and 6b; Table V). McAo, i.e., an isometric contraction

Table III. Time of Mitral Valve Opening in Cases of Mitral Valvular Discase.

\begin{tabular}{l|c|c|c}
\hline IIMO & MS & MSI & Healthy \\
\hline $0.02^{\text {sec. }}$ & $3^{\text {cases }}$ & $4^{\text {cases }}$ & cases \\
0.03 & 9 & 2 & 0 \\
0.04 & 7 & 5 & 0 \\
0.05 & 3 & 3 & 10 \\
0.06 & 8 & 6 & 23 \\
0.07 & 0 & 1 & 28 \\
0.08 & 0 & 1 & 9 \\
0.09 & 0 & 0 & 9 \\
0.10 & 0 & 0 & 2 \\
& cases & cases & cases \\
\hline
\end{tabular}

Table IV. Time of Pulmonic Valve Opening in Cases of Mitral Valvular Disease.

\begin{tabular}{l|c|c|c}
\hline QPo! & MS & MSI & Healthy \\
\hline sec. & $0^{\text {cases }}$ & 1 & cases \\
0.08 & 1 & 1 & 0 \\
0.09 & 5 & 3 & 5 \\
0.10 & 5 & 3 & 7 \\
0.11 & 5 & 4 & 8 \\
0.12 & 4 & 1 & 4 \\
0.13 & 0 & 2 & 1 \\
0.14 & 0 & 1 & 0 \\
0.15 & 0 & 0 & 0 \\
0.16 & 1 & 0 & 0 \\
0.17 & cases & 0 \\
\hline & 21 & 16 & cases \\
\end{tabular}




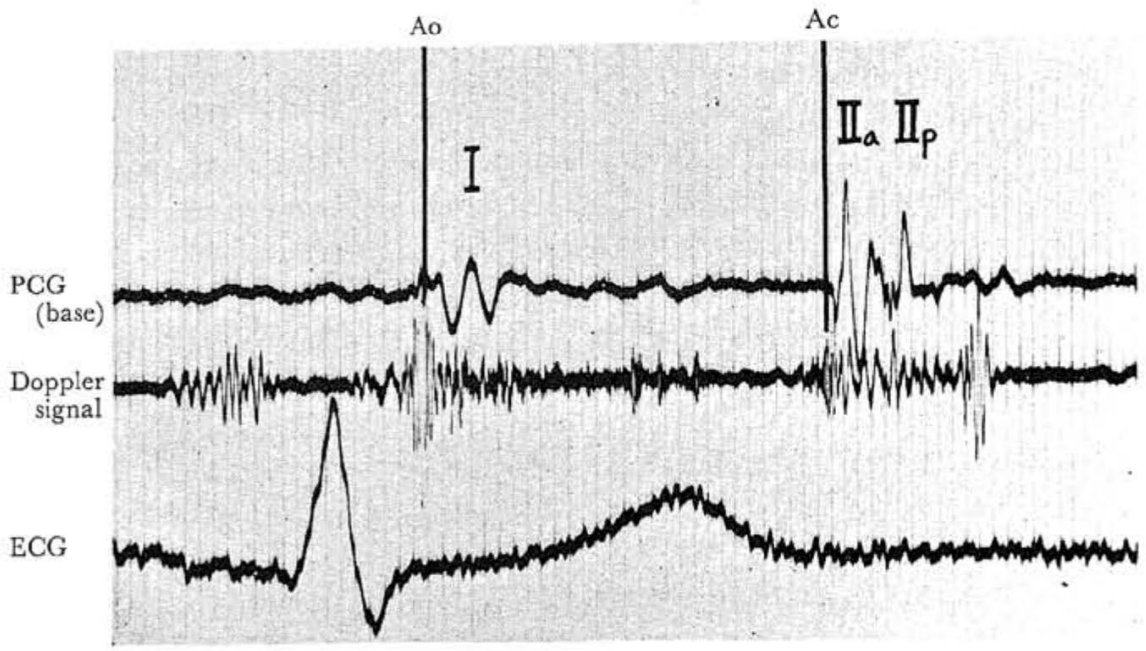

a

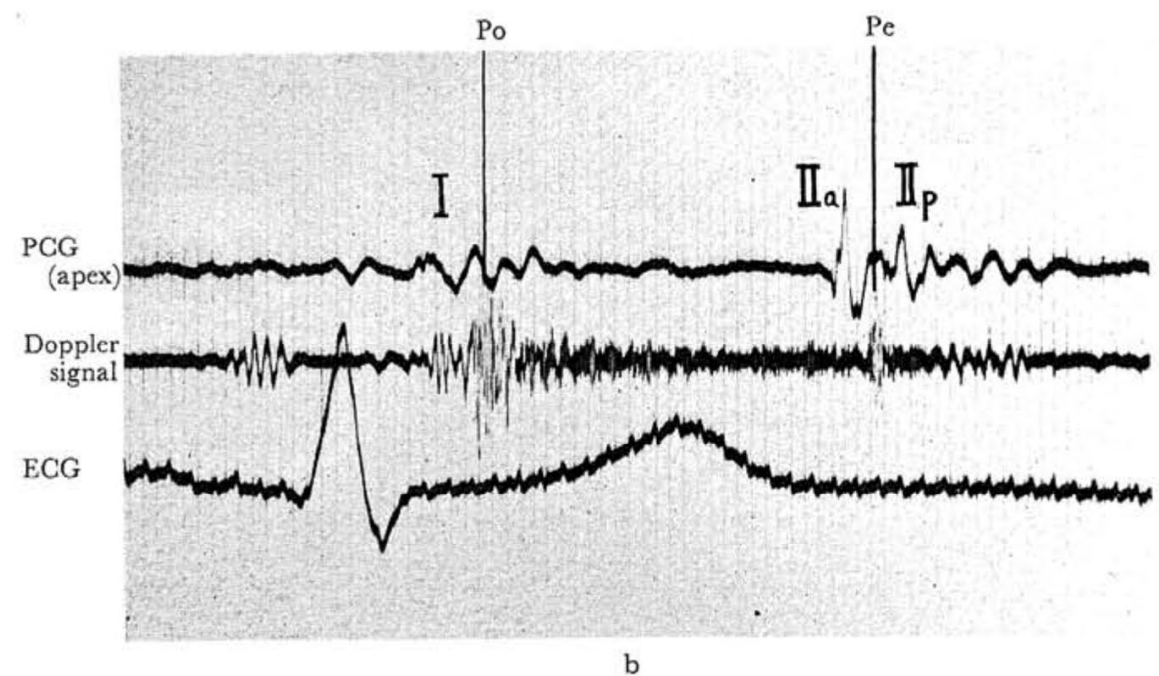

Fig. 6. Movements of semilunar valves in mitral stenosis.

a, aortic valve. b, pulmonic valve. Ao precedes Po.

(K.H., a 37-year-old man with mitral stenosis)

of the left ventricle, was markedly shorter than that of the right ventricle, TcPo, in cases of mitral stenosis.

For instance, in cases of mitral valve, the valves move in early systole in the order of Tc, Mc, Ao and Po. An isometric contraction and isometric relaxation of the left ventricle are shorter than those of right ventricle. 
Table V. Time of Semilunar Valve Opening in Cases Showing Identification of Both Ao and Po.

\begin{tabular}{l|c|c|c|c|c|c|c|c|c}
\hline Patient & Age & Sex & $\begin{array}{c}\text { Clinical } \\
\text { Diagnosis }\end{array}$ & QMc & QTc & QAo & QPo & McAo & TcPo \\
\hline & & & & sec. & sec. & sec. & scc. & sec. & sec. \\
S. K. & 26 & $\mathrm{f}$ & MS & 0.07 & 0.05 & 0.09 & 0.12 & 0.02 & 0.05 \\
K. T. & 38 & $\mathrm{f}$ & MS & 0.09 & 0.08 & 0.11 & 0.12 & 0.02 & 0.04 \\
K. H. & 37 & m & MS & 0.07 & 0.06 & 0.09 & 0.12 & 0.02 & 0.06 \\
Y. K. & 24 & $\mathrm{f}$ & MSI & 0.06 & 0.05 & 0.11 & 0.10 & 0.05 & 0.05 \\
M. K. & 23 & $\mathrm{f}$ & MSI & 0.08 & 0.04 & 0.11 & 0.15 & 0.03 & 0.11 \\
M. K. & 25 & m & MSI & 0.08 & 0.06 & 0.13 & 0.12 & 0.05 & 0.06 \\
Y. T. & 12 & $\mathrm{f}$ & MSI & 0.04 & - & 0.08 & 0.10 & 0.04 & - \\
& & & & & & & & &
\end{tabular}

\section{Discussion}

In most cases of mitral valvular disease of the heart, signal Mps is absent. It is suggested that the mitral valve does not move when the blood is impelled into the left ventricle by means of the atrial contraction. Here a limitation of movability of the mitral valve is assumed. In the meantime, Tps appears. In the early stage of the study with the ultrasonic Doppler method, these findings afforded an influential approach to the determination of the detecting area for the mitral valve and the tricuspid valve.

In healthy subjects the tricuspid valve closure is about the same as the mitral valve closure or 0.01-0.02 sec. earlier than the latter ${ }^{1), 61,7)}$ (Fig. 1c and 1d). But it seems that the tricuspid valve closure definitely precedes the mitral valve closure in cases of mitral valvular disease. This is mainly due to a delay in the mitral valve closure. Here a delay in the mitral valve closure seems to be related with a delay in the onset of the apical first sound ${ }^{8)-13)}$ in cases of mitral stenosis. The development of the delay seems to be due to such factors as an elevation in left atrial pressure, a hardness of the mitral valve, an insufficient left ventricular filling, and a leakage of pressure due to a regurgitation through the mitral ostium. The latter factor, however, does not seem to be so important, since many cases with mitral regurgitation have been encountered which showed no delay of Mc. Julian ${ }^{10)}$ stated that cases of mitral stenosis complicated with aortic regurgitation might show no delay in the mitral valve closure. The cases of mitral stenosis and insufficiency in the present study showed only a slight delay in the mitral valve closure. This no or slight delay may be attributable to a trend of increase in the diastolic filling of the left ventricle.

The tricuspid valve closure in some cases showed a more or less delay in comparison to the healthy subjects. But in cases of hypertension with a pressure overloading on the left ventricle, the mitral valve closure showed no delay, and showed a tendency to precede the tricuspid valve closure. ${ }^{14)}$ Thus it is not assumed that cases of mitral valvular disease with a pressure over- 
loading of the right ventricle may develop a delay in the closure of the tricuspid valve. However, there has been also a report ${ }^{15}$ that cases of hypertension might develop a delay in the mitral valve closing time. Much seems left to be studied concerning the closing time of the atrioventricular valve in the ventricle which is overloaded with pressure.

An early appearance of the mitral valve opening, i.e., a shortening of IIMo constitutes another feature in mitral valvular disease. Mitral opening snap begins nearly at the end of signal Mo. It is confirmed that mitral snap is referable to the mitral valve opening. Mitral snap has been understood to approach the second heart sound more as a pulmonary wedge pressure is more elevated. ${ }^{101,11), 16), 17)}$ So far as mitral stenosis is concerned, cases of marked right heart preponderance in ECG show a marked shortening of IIMo compared with cases without remarkable abnormality in ECG. ${ }^{1)}$ It is suggested that an elevation in left atrial pressure makes a main factor for the early development of Mo. As reported in our previous paper, ${ }^{1)}$ cases with an overloading on the left ventricle or myocardial changes show a delay in the development of Mo and prolongation of an isometric relaxation of the left ventricle. Thus it is interpreted that a less shortening of IIMo in cases of mitral stenosis and insufficiency than that in cases of mitral stenosis alone may be due to the effect of a left ventricular overloading. Another interpretation holds a fact that left atrial pressure in mitral stenosis and insufficiency is generally not so high as that in mitral stenosis may be a causative factor.

As mentioned above, cases with left ventricular overloading show a delay in the mitral valve opening, a prolongation of IIMo, and an appearance of Mo after To. ${ }^{1)}$ Thus, it is expected that cases of mitral valvular disease of the heart with a right ventricular overloading may develop a delay in the tricuspid opening. But, the development of To later than Mo in the present study seems mainly due to the early development of Mo. Contrary to our expectation, no prolongation of IITo seems to have developed because IITo (or IIpTo) is $0.04-0.07 \mathrm{sec}$. except for a case of IIpTo $=0.10 \mathrm{sec}$. The mechanism of the differences in the behaviors of the atrioventricular valves for the right and left ventricles is still unclarified. Cases with a left ventricular overloading having a tendency of heart failure develop a decrease in the prolongation

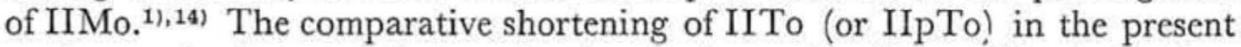
study may be due to such a mechanism as shows the aforesaid in the prolongation of IIMo.

Regarding healthy subjects, there still remains a discussion over the precedence of either Ao and Po. As reported already, ${ }^{1), 6)}$ the ultrasonic Doppler method has revealed that healthy subjects show that the aortic valve opening begins $0.09-0.14$ sec. $(0.107$ sec. ; 7 cases) after the beginning of the QRS and the pulmonary valve opening begins $0.09-0.13 \mathrm{sec}$. $(0.106 \mathrm{sec}$; 25 cases) afterward. But in cases where both signals (Ao and $\mathrm{Po}$ ) are recorded, Po preceded Ao by 0.02 sec. or less. Since an elevation in pulmonary artery pressure is present in cases of mitral valvular disease, a delay is expected in the 
opening of the pulmonary valve. The values described in Table V indicate that some cases of mitral valvular disease show a delay in the pulmonary valve opening which develops later than the opening of aortic valve. However, technically the values described in Table IV seem to have a possibility of introduction of real QAo. QAo in cases of mitral valvular disease seems to demonstrate similar values to that of healthy subjects.

It is revealed that the valves in early systole open and close in the order of Tc, Mc, Ao and Po. The results thus obtained are correspondent with those of $\mathrm{Ueda}^{13)}$ and his associates obtained with other methods.

\section{SUMMARY}

Studies were made with ultrasonic Doppler method of the time of valvular movements in cases of mitral valvular disease with sinus rhythm and the following results were obtained:

(1) Mps is absent, i.e. limitation of the movability of the mitral valve is suggested. The absence of Mps constitutes a diagnostic significance.

(2) Mitral valve closing develops later than that in healthy subjects.

(3) Pulmonary valve opening seems to develop later than that in healthy subjects.

(4) The valvular movements appear in early systole in the following order : tricuspid valve closing, mitral valve closing, aortic valve opening and pulmonary valve opening.

(5) Mitral valve opening appears early, prior to tricuspid valve opening.

(6) Mitral opening snap appears to begin in the last half of mitral valve opening.

(7) The isometric contraction and isometric relaxation of the left ventricle are shorter than those of the right ventricle.

(8) The time of tricuspid valve movements does not seem to show marked changes. This finding leaves room for further investigation.

(9) The above findings are generally more conspicuous in cases of mitral stenosis than in those of mitral stenosis and insufficiency.

(Published in Med. J. Osaka Univ. 11: 4549, 1959 in Japanese)

\section{REFERENCES}

1. Yoshida, T., Mori, M., Nimura, Y., Hikita, G., Takagishi, S., Nakanishi, K., and Satomura, S.: Am. Heart J. in the press.

2. Satomura, S.: J. Acoust. Soc. Am. 29 : 1181, 1957.

3. Yoshida, T., Mori, M., Nimura, Y., Takagishi, S., and Nakanishi, K.: Sogo Rinsho 8: 591, 1959 (In Japanese).

4. Yoshida, T., Mori, M., Nimura, Y., Hikita, G., and Satomura, S.: Respiration \& Circulation 7: 721, 1959 (In Japanese).

5. Edler, I. and Hertz, C. H. : Kungl. Fysiografiska Sällskapets I Lund Förhandlingar 24: Nr. 5, 1, 1954.

6. Takagishi, S.: Med. J. Osaka Univ. 11: 5317, 1959 (In Japanese). 
7. Yoshida, T., Mori, M., Nimura, Y., Takagishi, S., and Nakanishi, K.: Nippon Rinsho 17: 1441, 1959 (In Japanese).

8. Wells, B. : Brit. Heart J. 16: 261, 1954.

9. Kelly, J. J. : Am. J. Med. 19: 862, 1955.

10. Julian, D. and Davies, L. G.: Brit. Heart J. 19: 486, 1957.

11. Yamakawa, K., Yamamoto, T., Ohta, S., and Kitamura, K. : Jap. Circulation J. 18: 127, 1954 .(In Japanese).

12. Yamakawa, K.: Sinzo No Chosin, Igakushoin, Tokyo, 1956 (In Japanese).

13. Ueda, H., Yoshimura, S., Kaito, G., Sugai, J. Yamadera, T., Hara, Y., Hashimoto, H., Sato, Y., and Sato, F.: Jap. Circulation J. 21: 170, 1957 (In Japanese).

14. Nakanishi, K.: Med. J. Osaka Univ. 11: 4549, 1959 (In Japanese).

15. Weissler, A. M., Leonard, J. J., and Warrens, J. V.: Circulation 18: $165,1958$.

16. Ohta, S.: J. Jap. Soc. Int. Med. 46: 739, 1957 (In Japanese).

17. Bayer, O., Loogen, F., and Walter, H. H.: Am. Heart J. 51 : 234, 1956. 